\title{
Polyaniline-polypyrrole nanocomposites using a green and porous wood as support for supercapacitors
}

\author{
Jian LI (凶), Yue JIAO \\ Material Science and Engineering College, Northeast Forestry University, Harbin 150040, China
}

\begin{abstract}
Wood is an ideal type of support material whose porous structure and surface functional groups are beneficial for deposition of various guest substances for different applications. In this paper, wood is employed as a porous support, combined with two kinds of conductive polymers (i.e., polyaniline (PANI) and polypyrrole (PPy)) using an easy and fast liquid polymerization method. Scanning electron microscope observations indicate that the PANI-PPy complex consists of nanoparticles with a size of $\sim 20 \mathrm{~nm}$. The interactions between oxygencontaining groups of the wood and the nitrogen composition of PANI-PPy were verified by Fourier transform infrared spectroscopy. The self-supported PANI-PPy/ wood composite is capable of acting as a free-standing supercapacitor electrode, which delivers a high gravimetric specific capacitance of $360 \mathrm{~F} \cdot \mathrm{g}^{-1}$ at $0.2 \mathrm{~A} \cdot \mathrm{g}^{-1}$.
\end{abstract}

Keywords wood, polypyrrole, polyaniline, supercapacitors, nanocomposites

\section{Introduction}

Supercapacitors are a novel class of electrochemical energy storage systems, which have the ability to bridge the power/energy gap between traditional dielectric capacitors and batteries/fuel cells ${ }^{[1]}$. It is known that supercapacitors universally possess ultrahigh power densities $^{[2]}$, fast charge-discharge properties ${ }^{[3]}$, excellent reversibility ${ }^{[4]}$, a long lifecycle ${ }^{[5]}$ and wide operation temperature ${ }^{[6]}$. According to the difference in energy storage mechanisms, supercapacitors can be classified into two categories: electrical double-layer capacitor (EDLC) materials (such as carbon materials) and pseudocapacitive materials (e.g., transition-metal oxides or hydroxides and conductive polymers $)^{[7]}$. For EDLCs, the capacitance is due to electrostatic charge separation at the interface

Received January 9, 2019; accepted March 14, 2019

Correspondence: nefulijian@163.com between the electrode and the electrolyte; while for pseudocapacitors, the capacitance relies on fast and reversible faradaic redox reactions to store charges ${ }^{[8]}$. In general, pseudocapacitive materials deliver higher capacitance and energy density than those of EDLC materials. Conductive polymers (for instance polyaniline (PANI), polypyrrole (PPy) and polythiophene (PTh)) are a class of important pseudocapacitive materials ${ }^{[9]}$. Supercapacitor electrodes prepared with these conductive polymers have already exhibited numerous merits, such as high electrochemical activity and conductivity, good cost-effectiveness and easy of synthesis ${ }^{[10-13]}$. Nevertheless, because of their poor mechanical strength, difficulty in processing and handling, ease of agglomeration and low porosity, conductive polymers are widely combined with various host materials, e.g., carbon aerogels ${ }^{[14]}$, cellulose nanofibrils $^{[15]}$ and cellulose paper ${ }^{[16]}$.

Wood is a green and ideal matrix with many fascinating properties, for instance, renewability, low density and thermal expansion, ease of machining, and good mechanical strength. Wood has been combined with various organic or inorganic substances (such as $\mathrm{ZrO}_{2}{ }^{[17]}$, graphene $^{[18]}$ and polyvinyl alcohol ${ }^{[19]}$ ) for multifarious applications (such as ultraviolet resistant catalysts and reinforcing materials). In the field of supercapacitors, wood has abundant direct channels with low tortuosity along the tree-growing direction, which have been demonstrated to allow fast transport of electrolyte ions ${ }^{[20]}$. Moreover, in our previous research, we have integrated wood with PANI via a method of oxidative polymerization and this binary composite shows a moderate specific capacitance of $304 \mathrm{~F} \cdot \mathrm{g}^{-1}$ at $0.1 \mathrm{~A} \cdot \mathrm{g}^{-1}{ }^{[21]}$. However, the value can be further improved by introducing new electrochemically active components.

Herein, natural wood was used as a green and nanoporous support to combine with PANI-PPy nanocomposites by virtue of a liquid-phase synthesis method. The micromorphology and chemical bonds of the asprepared wood-supported PANI-PPy hybrid (coded as PANI-PPy/wood) was studied by scanning electron 
microscopy (SEM) and Fourier transform infrared spectrum (FTIR) analysis, respectively. In addition, the electrochemical performances of PANI-PPy/wood were studied in a three-electrode configuration via cyclic voltammetry $(\mathrm{CV})$, galvanostatic charge-discharge (GCD) and electrochemical impedance spectroscopy (EIS) methods.

\section{Materials and methods}

\subsection{Materials}

Pyrrole, iron (III) chloride hexahydrate $\left(\mathrm{FeCl}_{3} \cdot 6 \mathrm{H}_{2} \mathrm{O}\right)$, hydrochloric acid $(\mathrm{HCl}, 37 \%)$, aniline and ammonium peroxide sulfate, tert-butyl alcohol and absolute ethyl alcohol were supplied by Kemiou Chemical Reagent Co., Ltd. (China). Paulownia wood processing residues were collected from a wood-working factory in China (Linwei Wood Industry, Caoxian County in Shandong province) and cut into slices with a thickness of $\sim 1 \mathrm{~mm}$. These wood slices were rinsed ultrasonically with absolute ethyl alcohol and distilled water for $30 \mathrm{~min}$ and dried at $60^{\circ} \mathrm{C}$ for $24 \mathrm{~h}$.

\subsection{Deposition of PANI on wood support}

The deposition of PANI on the wood support was performed by the method described $i^{[21]}$. Briefly, the wood slices were immersed in a $100 \mathrm{~mL}$ aqueous solution of aniline monomer for $24 \mathrm{~h}$ at room temperature. The mass ratio of wood to aniline was set as 1:10. Subsequently, a mixture of oxidant (ammonium peroxide sulfate, $0.08 \mathrm{~mol}$ ) and dopant (hydrochloric acid, $1 \mathrm{~mol} \cdot \mathrm{L}^{-1}, 96 \mathrm{~mL}$ ) was dropwise added into the above solution precooled to $0^{\circ} \mathrm{C}$. The reaction was allowed to proceed for $4 \mathrm{~h}$ at $0-10^{\circ} \mathrm{C}$. Finally, the resultant PANI/ wood slice was rinsed with a large amount of distilled water.

\subsection{Deposition of PPy on PANI/wood}

The deposition of PPy on PANI/wood was conducted according to the method in our previous report ${ }^{[11]}$. Typically, PANI/wood was soaked in $100 \mathrm{~mL}$ water solution of pyrrole monomer $\left(1.35 \mathrm{~mol} \cdot \mathrm{L}^{-1}\right)$ for $2 \mathrm{~h}$ and then slowly mixed with an aqueous solution of $\mathrm{FeCl}_{3} \cdot 6 \mathrm{H}_{2} \mathrm{O}$ (oxidant). The molar ratio of ferric iron/ pyrrole was set as 1:1. After the polymerization, the asprepared PANI-PPy/wood was washed with distilled water and tert-butyl alcohol in sequence and then underwent a freeze-drying process for $24 \mathrm{~h}$ at $25 \mathrm{~Pa}$.

\subsection{Characterization}

The morphology was characterized by an SEM (Hitachi, S4800) equipped with an energy dispersive X-ray (EDX) detector for elemental analysis. FTIR spectra were recorded using a Nicolet Nexus 670 FTIR instrument in the range of $500-4000 \mathrm{~cm}^{-1}$ with a resolution of $4 \mathrm{~cm}^{-1}$. Electrochemical tests were conducted in a three-electrode setup with PANI-PPy/wood as the working electrode and an $\mathrm{Ag} / \mathrm{AgCl}$ electrode and a Pt wire electrode as reference and counter electrodes, respectively and $1 \mathrm{~mol} \cdot \mathrm{L}^{-1} \mathrm{HCl}$ aqueous solution electrolyte. CV, GCD and EIS tests were implemented using a CS350 electrochemical workstation (Wuhan CorrTest Instruments Co., Ltd). CV tests were conducted over the potential window from $-0.2 \mathrm{~V}$ to $1.0 \mathrm{~V}$ at different scan rates of $5,10,20$ and $50 \mathrm{mV} \cdot \mathrm{s}^{-1}$. GCD curves were tested in the potential range of $0-0.8 \mathrm{~V}$ at different current densities of $0.2,0.5,1$ and $2 \mathrm{~A} \cdot \mathrm{g}^{-1}$. EIS measurements were carried out in the frequency range of $10^{5}$ to $0.01 \mathrm{~Hz}$ with an alternate current amplitude of $5 \mathrm{mV}$.

\section{Results and discussion}

\subsection{Schematic diagram for the synthesis of PANI-PPy/ wood}

Wood is a good porous support material for the growth of various guest substances. The abundant oxygen-containing functional groups (such as hydroxyl groups and carboxyl groups) on its surface have the ability to fix ions and small molecules and guide their in situ growth or polymerization. In this paper, based on this strategy, we used wood as host material; through a facile liquid polymerization approach, the PANI and PPy nanoparticles were successively deposited onto the surface of the wood matrix, resulting in the generation of a self-supported ternary composite, i.e., PANI-PPy/wood (Fig. 1).

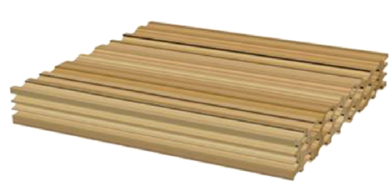

Wood support
Deposition of PANI

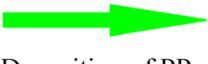

Deposition of PPy

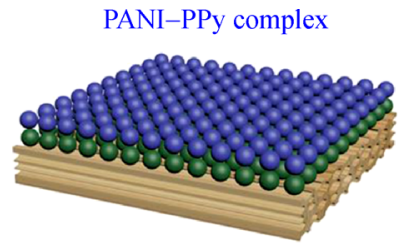

PANI-PPy/wood

Fig. 1 Schematic diagram for the synthesis of PANI-PPy/wood 


\subsection{Micromorphology and elemental analysis}

The micromorphology changes in the wood support before and after the deposition of PANI and PPy were studied by SEM observations. The structure of wood is presented in Fig. 2(a), where wood vessels can be clearly identified. After the deposition of PANI and PPy, the characteristic structure of wood disappeared; instead, a large number of nano-scale particles appeared on the surface of the wood support (Fig. 2(b)). Based on the higher-magnification SEM image in Fig. 2(c), we found that these nanoparticles have a size of $\sim 20 \mathrm{~nm}$. The tight connection of these particles is expected to improve the structural stability of the composite during the charge-discharge process. Also, this connection may be beneficial for the transport of electrons. The elemental compositions of the wood and PANI-PPy/wood were investigated by EDX analysis. As seen in Fig. 2(d), the elements including C, $\mathrm{O}$ and Au were detected in the wood. The Au element in particular came from the coating layer used for electric conduction during the SEM observation. For the PANI-PPy/wood, a new N element signal was detected derived from the PANI and $\mathrm{PPy}$ and a $\mathrm{Cl}$ signal derived from the reactants $\left(\mathrm{FeCl}_{3}\right.$ or and $\mathrm{HCl}$ ).

\subsection{FTIR analysis}

The chemical composition of the wood support and PANI-PPy/wood were studied by FTIR analysis. As seen in Fig. 3, for the wood support, the wide band at 2990$3720 \mathrm{~cm}^{-1}$ is attributed to the $\mathrm{O}-\mathrm{H}$ stretching of polymeric compounds, while the bands at 2920 and $2857 \mathrm{~cm}^{-1}$ are derived from the alkane $\mathrm{C}-\mathrm{H}$ asymmetric and symmetric stretching vibrations ${ }^{[22]}$, respectively. The band at $1734 \mathrm{~cm}^{-1}$ belongs to the $\mathrm{C}=\mathrm{O}$ stretching in unconjugated ketone, carbonyl and aliphatic groups (xylan) and the strong band at $1641 \mathrm{~cm}^{-1}$ is due to the bending mode of the absorbed water ${ }^{[23]}$. The bands at 1420, 1163 and $1066 \mathrm{~cm}^{-1}$ are attributed to the cellulose structure, correspond to the $\mathrm{CH}_{2}$ symmetric bending, $\mathrm{C}-\mathrm{O}$ antisymmetric stretching and $\mathrm{C}-\mathrm{O}-\mathrm{C}$ pyranose ring skeletal vibration ${ }^{[24]}$, respectively. The band at $1261 \mathrm{~cm}^{-1}$ originates from the $\mathrm{C}-\mathrm{O}$ symmetric stretching.

For PANI-PPy/wood, the band position of the O-H stretching moves to the lower wavenumber from $3441 \mathrm{~cm}^{-1}$ compared to $3423 \mathrm{~cm}^{-1}$ in wood, possibly due to the interaction between the oxygen-containing groups of wood and the nitrogen composition of PANI-PPy. The strong interaction between them helps to enhance the adhesion effect of active materials on the wood, possibly contributing to the strengthening of electrochemical stability. The bands at $1541 \mathrm{~cm}^{-1}$ and $1458 \mathrm{~cm}^{-1}$ may be assigned to the $\mathrm{C}-\mathrm{C}$ and $\mathrm{C}-\mathrm{N}$ stretching vibrations in the pyrrole ring, respectively. In addition, the bands at $1031 \mathrm{~cm}^{-1}$ and $774 \mathrm{~cm}^{-1}$ are due to the $\mathrm{N}-\mathrm{H}$ in-plane vibration of the $\mathrm{PPy}$ ring and $\mathrm{C}-\mathrm{H}$ out-of-plane ring deformation $^{[25]}$, respectively. Additionally, the $\mathrm{C}-\mathrm{H}$ stretching vibration of aromatic conjugation of PANI (a)

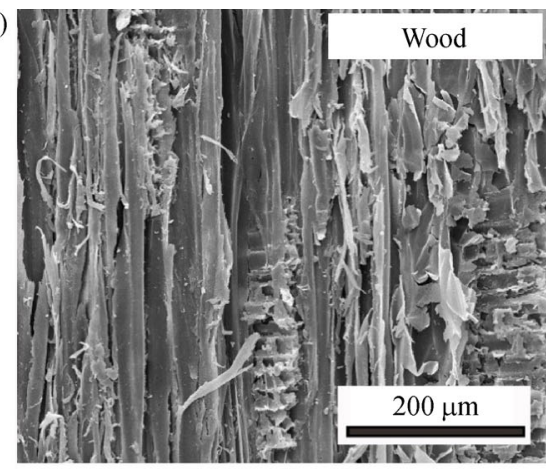

(c)

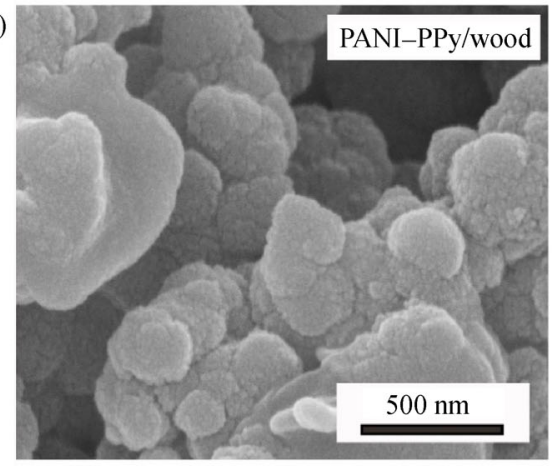

(b)

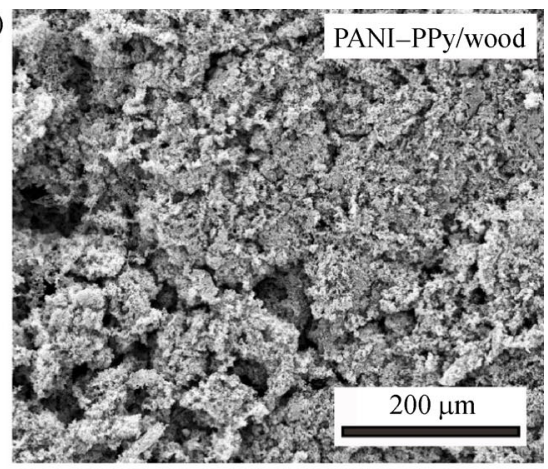

(d)

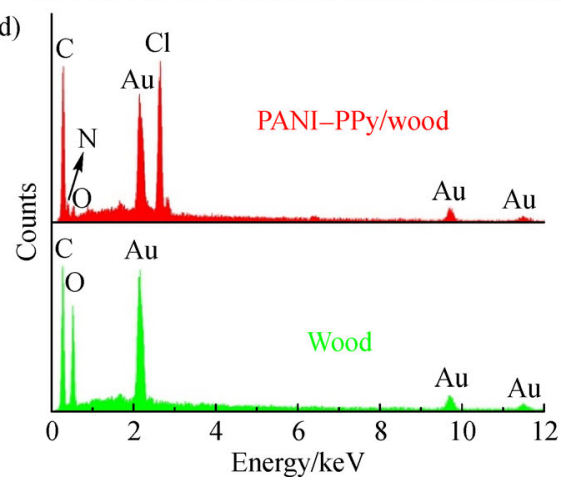

Fig. 2 SEM images of the wood support (a) and PANI-PPy/wood (b, c); (d) EDX patterns of the wood support and PANI-PPy/wood. 
displays adsorption bands at $1375 \mathrm{~cm}^{-1}$ and $1163 \mathrm{~cm}^{-1[26]}$. Moreover, the band at $1298 \mathrm{~cm}^{-1}$ arises from the $\mathrm{C}-\mathrm{N}$ in-plane ring bending mode of PANI. The results of FTIR analysis thus suggest the successful synthesis of PANI and PPy on the surface of the wood host.

\subsection{Electrochemical analysis}

The PANI-PPy/wood can serve as a free-standing electrode and its electrochemical properties were studied by CV, GCD and EIS in a three-electrode system, using a $1 \mathrm{~mol} \cdot \mathrm{L}^{-1} \mathrm{HCl}$ aqueous solution. The $\mathrm{CV}$ curves of the PANI-PPy/wood electrode at different scan rates are displayed in Fig. 4. At scan rates of 5 and $10 \mathrm{mV} \cdot \mathrm{s}^{-1}$, the $\mathrm{CV}$ plots display quasi-rectangular shapes, whereas when the scan rate reached $20 \mathrm{mV} \cdot \mathrm{s}^{-1}$ and above, the $\mathrm{CV}$ curves became shuttle-like in shape, which can possibly be attributed to the slower entering/ejecting and diffusion rates of counterions compared to the transfer rate of electrons in the electrode at the high scan rates ${ }^{[11]}$.

According to the GCD traces (Fig. 5) at different current densities, the obvious curvature reflects the process of a faradic reaction. In addition, during the charging and discharging step, the charge curves of the PANI-PPy/wood electrode are almost symmetric to their corresponding discharge counterparts, suggesting good capacitive behavior and highly reversible electrochemical reactions. Moreover, GCD is a conventional method to calculate the capacitance value of supercapacitor electrodes. The gravimetric $\left(C_{m}, \mathrm{~F} \cdot \mathrm{g}^{-1}\right)$ specific capacitances were calculated from these GCD curves at different current densities based on the following equations ${ }^{[27]}$ :

$$
C_{m}=I \Delta t / m \Delta V
$$

where $I(\mathrm{~A})$ is the discharge current, $\Delta t(\mathrm{~s})$ is the discharge time, $m(\mathrm{~g})$ is the mass of active materials (the areal density of PANI-PPy on the electrode is ca. $10 \mathrm{mg} \cdot \mathrm{cm}^{-2}$ ) and $\Delta V$ $(\mathrm{V})$ is the operation discharge voltage window. The result displays that the PANI-PPy/wood electrode achieves the highest value of $360 \mathrm{~F} \cdot \mathrm{g}^{-1}$ at $0.2 \mathrm{~A} \cdot \mathrm{g}^{-1}$, which is higher than that of some congeneric products in the literature, such as core-sheath structured bacterial cellulose/polypyrrole nanocomposites $\left(316 \mathrm{~F} \cdot \mathrm{g}^{-1} \text { at } 0.2 \mathrm{~A} \cdot \mathrm{g}^{-1}\right)^{[28]}$,

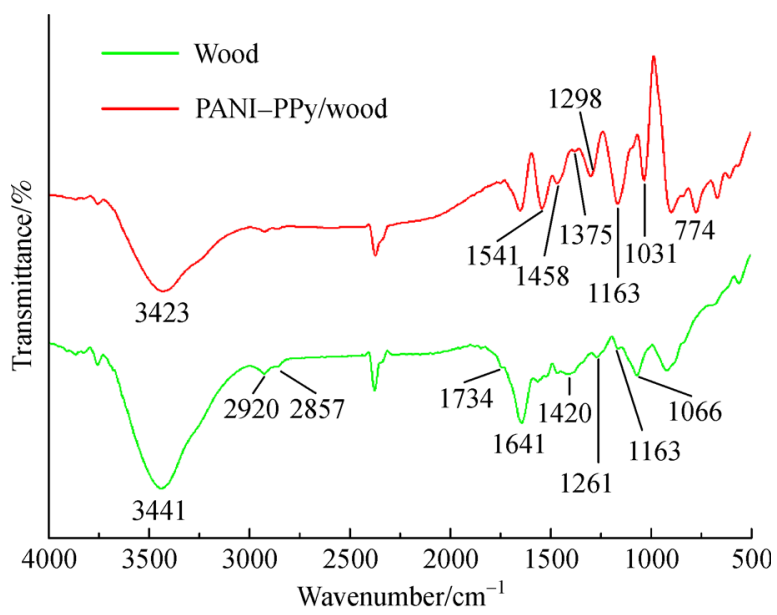

Fig. 3 FTIR spectra of the wood support and PANI-PPy/wood

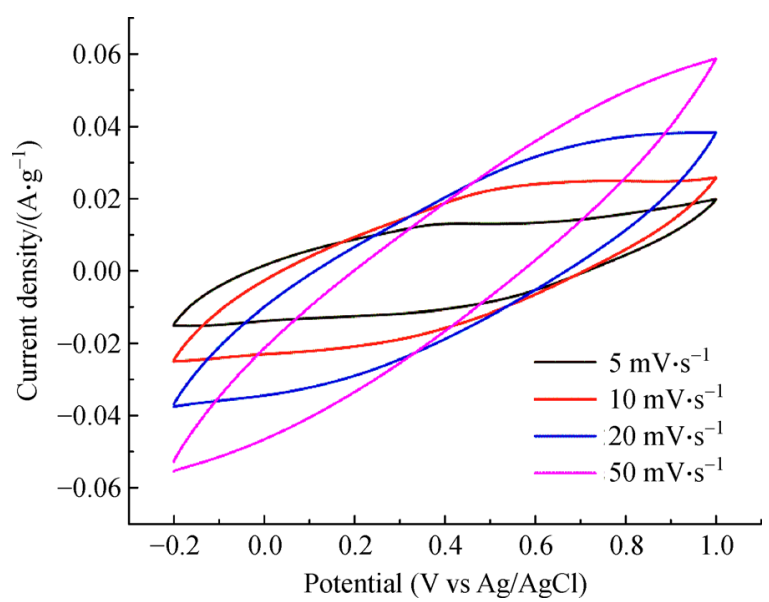

Fig. 4 CV curves of the PANI-PPy/wood electrode at different scan rates 
bacterial cellulose nanofiber-supported polyaniline nanocomposites $\left(273 \mathrm{~F} \cdot \mathrm{g}^{-1} \text { at } 0.2 \mathrm{~A} \cdot \mathrm{g}^{-1}\right)^{[29]}$ and PANI/wood $\left(280 \mathrm{~F} \cdot \mathrm{g}^{-1} \text { at } 0.2 \mathrm{~A} \cdot \mathrm{g}^{-1}\right)^{[21]}$. With the increase of current densities from 0.5 to $2.0 \mathrm{~A} \cdot \mathrm{g}^{-1}$, the specific capacitance value decreased to 259,180 and $77 \mathrm{~F} \cdot \mathrm{g}^{-1}$, as shown in Fig. 6. The decline is associated with the low utilization of electroactive materials at high discharge current densities since the electrolyte ions cannot enter into the inner structure of the active material, and only the outer active surface was utilized for charge storage ${ }^{[30]}$.

EIS was conducted to evaluate the charge transfer and electrolyte diffusion in the electrode/electrolyte interface. Figure 7 shows the Nyquist plot for the PANI-PPy/wood electrode. As illustrated, the curve has a characteristic semicircle over the high frequency range, a $45^{\circ}$ Warburg region, and a linear pure capacitor part in the low frequency region. In the high-frequency region, the intersection of the semicircle with the real axis reveals the solution resistance value (i.e., $1.1 \Omega$ ), indicative of a high electrical conductivity of the electrolyte. The diameter of the semicircle represents the charge transport resistance (ca. $6.6 \Omega$ ). In the medium-frequency region, the projected length of the $45^{\circ}$ Warburg region on the real impedance axis characterizes the ion penetration process. This short Warburg length indicates a short ion-diffusion path. In the low frequency region, the slope of the linear part is low, suggesting a diffusion-controlled behavior ${ }^{[31]}$.

\section{Conclusions}

The electrochemically active substances, PANI and PPy, were deposited in sequence onto the surface of a wood support using a liquid-phase polymerization method. The PANI-PPy mixed particles have a diameter of around $20 \mathrm{~nm}$ according to SEM observations. EDX and FTIR analysis also reflects the characteristics of both PANI and PPy compositions and demonstrates their successful synthesis on the wood surface. Furthermore, this selfsupported PANI-PPy/wood composite has the ability to serve as a free-standing electrode, which shows a high gravimetric specific capacitance of $360 \mathrm{~F} \cdot \mathrm{g}^{-1}$ at $0.2 \mathrm{~A} \cdot \mathrm{g}^{-1}$ superior to some congeneric products.

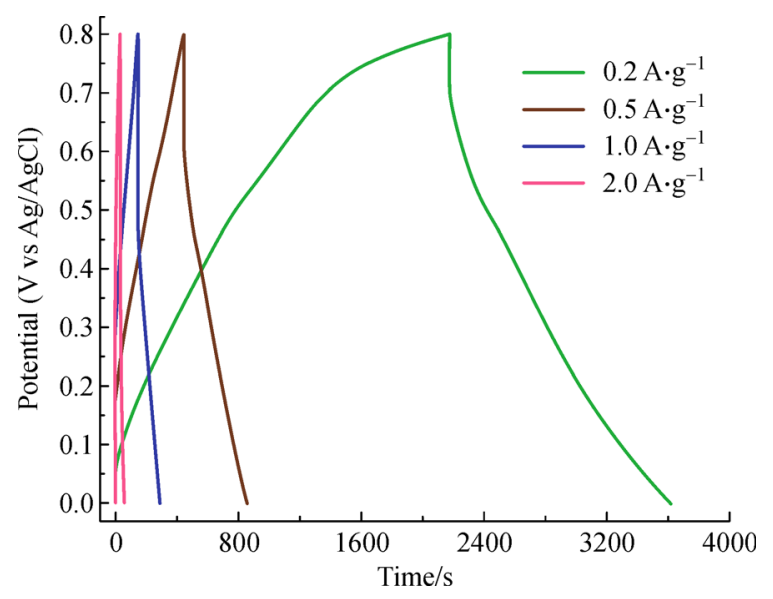

Fig. 5 GCD curves of the PANI-PPy/wood electrode at different current densities

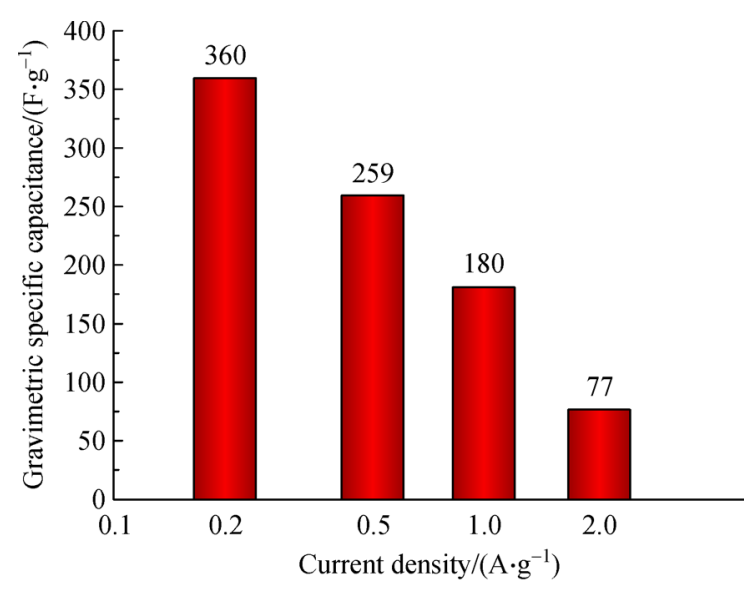

Fig. 6 Gravimetric specific capacitances of the PANI-PPy/wood electrode at different current densities 


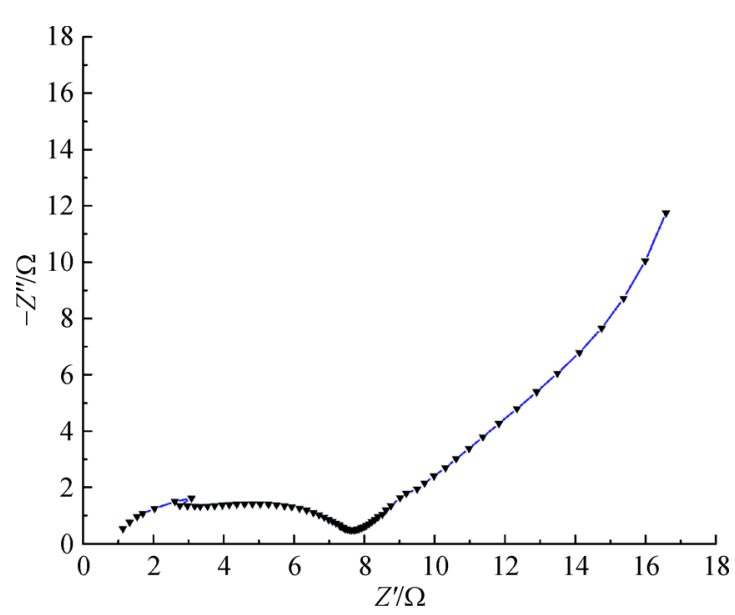

Fig. 7 Nyquist plots of the PANI-PPy/wood electrode

Acknowledgements This study was supported by the Fundamental Research Funds for the Central Universities (2572018AB09) and the National Natural Science Foundation of China (31470584).

Compliance with ethics guidelines Jian Li and Yue Jiao declare that they have no conflicts of interest or financial conflicts to disclose.

This article does not contain any studies with human or animal subjects performed by the any of the authors.

\section{References}

1. Wang G, Zhang L, Zhang J. A review of electrode materials for electrochemical supercapacitors. Chemical Society Reviews, 2012, 41(2): 797-828

2. Du C, Pan N. High power density supercapacitor electrodes of carbon nanotube films by electrophoretic deposition. Nanotechnology, 2006, 17(21): 5314-5318

3. Yan J, Fan Z, Wei T, Qian W, Zhang M, Wei F. Fast and reversible surface redox reaction of graphene- $\mathrm{MnO}_{2}$ composites as supercapacitor electrodes. Carbon, 2010, 48(13): 3825-3833

4. Hu C C, Chen J C, Chang K H. Cathodic deposition of $\mathrm{Ni}(\mathrm{OH})_{2}$ and $\mathrm{Co}(\mathrm{OH})_{2}$ for asymmetric supercapacitors: importance of the electrochemical reversibility of redox couples. Journal of Power Sources, 2013, 221: 128-133

5. Wan C, Jiao Y, Li J. A cellulose fibers-supported hierarchical forestlike cuprous oxide/copper array architecture as a flexible and freestanding electrode for symmetric supercapacitors. Journal of Materials Chemistry A: Materials for Energy and Sustainability, 2017, 5(33): 17267-17278

6. Hung K, Masarapu C, Ko T, Wei B. Wide-temperature range operation supercapacitors from nanostructured activated carbon fabric. Journal of Power Sources, 2009, 193(2): 944-949

7. Zhi M, Xiang C, Li J, Li M, Wu N. Nanostructured carbon-metal oxide composite electrodes for supercapacitors: a review. Nanoscale, 2013, 5(1): 72-88

8. Zhong C, Deng Y, Hu W, Qiao J, Zhang L, Zhang J, Qiao J, Zhang L, Zhang J. A review of electrolyte materials and compositions for electrochemical supercapacitors. Chemical Society Reviews, 2015, 44(21): 7484-7539

9. Snook G A, Kao P, Best A S. Conducting-polymer-based supercapacitor devices and electrodes. Journal of Power Sources, 2011, 196(1): 1-12

10. Zhang J, Zhao X S. Conducting polymers directly coated on reduced graphene oxide sheets as high-performance supercapacitor electrodes. Journal of Physical Chemistry C, 2012, 116(9): 5420-5426

11. Wan C, Jiao Y, Li J. Flexible, highly conductive, and free-standing reduced graphene oxide/polypyrrole/cellulose hybrid papers for supercapacitor electrodes. Journal of Materials Chemistry A: Materials for Energy and Sustainability, 2017, 5(8): 3819-3831

12. Wan C, Li J. Wood-derived biochar supported polypyrrole nanoparticles as a free-standing supercapacitor electrode. RSC Advances, 2016, 6(89): 86006-86011

13. Li J, Lu W, Yan Y, Chou T W. High performance solid-state flexible supercapacitor based on $\mathrm{Fe}_{3} \mathrm{O}_{4} /$ carbon nanotube/polyaniline ternary films. Journal of Materials Chemistry A: Materials for Energy and Sustainability, 2017, 5(22): 11271-11277

14. Wan C, Li J. Synthesis and electromagnetic interference shielding of cellulose-derived carbon aerogels functionalized with $\alpha-\mathrm{Fe}_{2} \mathrm{O}_{3}$ and polypyrrole. Carbohydrate Polymers, 2017, 161: 158-165

15. Tian J, Peng D, Wu X, Li W, Deng H, Liu S. Electrodeposition of Ag nanoparticles on conductive polyaniline/cellulose aerogels with increased synergistic effect for energy storage. Carbohydrate Polymers, 2017, 156: 19-25

16. Zhang Y Z, Wang Y, Cheng T, Lai W Y, Pang H, Huang W. Flexible supercapacitors based on paper substrates: a new paradigm for low-cost energy storage. Chemical Society Reviews, 2015, 44 (15): 5181-5199

17. Wan C, Lu Y, Sun Q, Li J. Hydrothermal synthesis of zirconium dioxide coating on the surface of wood with improved UV resistance. Applied Surface Science, 2014, 321: 38-42

18. Wan C, Jiao Y, Li J. In situ deposition of graphene nanosheets on wood surface by one-pot hydrothermal method for enhanced UVresistant ability. Applied Surface Science, 2015, 347: 891-897

19. Bana R, Banthia A K. Green composites: development of poly(vinyl alcohol)-wood dust composites. Polymer-Plastics Technology and Engineering, 2007, 46(9): 821-829

20. Chen C, Zhang Y, Li Y, Dai J, Song J, Yao Y, Gong Y, Kierzewski I, Xie J, Hu L. All-wood, low tortuosity, aqueous, biodegradable supercapacitors with ultra-high capacitance. Energy \& Environmental Science, 2017, 10(2): 538-545

21. Jiao Y, Wan C, Li J. Scalable synthesis and characterization of freestanding supercapacitor electrode using natural wood as a green substrate to support rod-shaped polyaniline. Journal of Materials Science Materials in Electronics, 2017, 28(3): 2634-2641

22. Wan C, Lu Y, Jiao Y, Jin C, Sun Q, Li J. Ultralight and hydrophobic nanofibrillated cellulose aerogels from coconut shell with ultrastrong adsorption properties. Journal of Applied Polymer Science, 2015, 132(24): 42037

23. Tjeerdsma B F, Militz H. Chemical changes in hydrothermal treated wood: FTIR analysis of combined hydrothermal and dry heattreated wood. Holz als Roh- und Werkstoff, 2005, 63(2): 102-111

24. Oh S Y, Yoo D I, Shin Y, Seo G. FTIR analysis of cellulose treated with sodium hydroxide and carbon dioxide. Carbohydrate 
Research, 2005, 340(3): 417-428

25. Xu J, Zhu L, Bai Z, Liang G, Liu L, Fang D, Xu W. Conductive polypyrrole-bacterial cellulose nanocomposite membranes as flexible supercapacitor electrode. Organic Electronics, 2013, 14 (12): 3331-3338

26. Xu J, Zhang Y, Zhang D, Tang Y, Cang H. Electrosynthesis of PANI/PPy coatings doped by phosphotungstate on mild steel and their corrosion resistances. Progress in Organic Coatings, 2015, 88: 84-91

27. Wan C, Yue J, Jian L. Core-shell composite of wood-derived biochar supported $\mathrm{MnO}_{2}$ nanosheets for supercapacitor applications. RSC Advances, 2016, 6(69): 64811-64817

28. Wang H, Bian L, Zhou P, Tang J, Tang W. Core-sheath structured bacterial cellulose/polypyrrole nanocomposites with excellent conductivity as supercapacitors. Journal of Materials Chemistry A: Materials for Energy and Sustainability, 2013, 1(3): 578-584

29. Wang H, Zhu E, Yang J, Zhou P, Sun D, Tang W. Bacterial cellulose nanofiber-supported polyaniline nanocomposites with flake-shaped morphology as supercapacitor electrodes. Journal of Physical Chemistry C, 2012, 116(24): 13013-13019

30. Chen H, Jiang J, Zhang L, Wan H, Qi T, Xia D. Highly conductive $\mathrm{NiCo}_{2} \mathrm{~S}_{4}$ urchin-like nanostructures for high-rate pseudocapacitors. Nanoscale, 2013, 5(19): 8879-8883

31. Zhao T, Jiang H, Ma J. Surfactant-assisted electrochemical deposition of $\alpha$-cobalt hydroxide for supercapacitors. Journal of Power Sources, 2011, 196(2): 860-864 